\title{
Pitfalls of Social Media in the Era of COVID-19 Pandemics
}

\author{
Abdullah ALANAZI ${ }^{\mathrm{a}}$, Hanan ALDOSARI ${ }^{\mathrm{a}}$ and Bakheet ALDOSARI ${ }^{\mathrm{a}, 1}$ \\ ${ }^{a}$ College of Public Health and Health Informatics, King Saud bin Abdulaziz University \\ for Health Sciences, Riyadh, Saudi Arabia
}

\begin{abstract}
In December 2019, Wuhan, China, reported an outbreak of nSARSCoV2 that caused viral pneumonia, COVID-19. Li Wenliang, a Chinese ophthalmologist, first communicated on Chinese social media about the existence and spread of the unknown viral pneumonia in Wuhan, China. By the end of March 2020, the virus had spread worldwide. However, non-scientific information related to the viral outbreak, disease, and mortality spread even faster on social media. This study performed literature searches among different databases, i.e., PubMed, PubMed Central, and Web of Science, to understand the pitfall of social media during the COVID-19 pandemic and the impact of non-scientific information on public health. Social media not only shared information regarding the outbreak of nSARS-CoV2 and COVID-19 disease but also misinformation regarding epidemiology, government policies, prevention, cure, and vaccination. Thus, strict regulation is required to control the spread of misleading information.
\end{abstract}

Keywords. COVID-19, nSARS-CoV2, social media, pitfalls, and falsification

\section{Introduction}

The outbreak of beta coronavirus in Wuhan, China, in December 2019, resulted in a global pandemic named COVID-19, affecting more than 150 million people with more than 3 million deaths so far and the world is still fighting to find a cure. COVID-19 vaccines received mixed responses resulting in vaccine hesitancy with social media playing a critical role in spreading information (generally fabricated) towards safety and efficacy.

Last year, several regions reported multiple waves of infection, and India being the worst hit. Platforms such as Facebook, YouTube, WhatsApp, Instagram, and Twitter, are the most widely used, and never before in human history, the sharing of information was so easily possible [1]. However, these social media platforms have shown a dual effect on human life. On the one hand, they have spread awareness and shared correct information, enabling many populations to adopt recommended precautions against the current infection pandemic [2]. On the other hand, there is the dark side of social media as well, and in the present pandemic, it has created a panic situation in every aspect of COVID-19 related to cause, spread, treatment, and vaccine.

It is essential to understand that more than 3 billion social media users worldwide remain active in sharing information, particularly non-scientific knowledge [3]. In India

${ }^{1}$ Corresponding Author, Bakheet ALDOSARI, College of Public Health \& Health Informatics, King Saud Bin Abdul-Aziz University for Health Sciences, Saudi Arabia. Email: dosarib@gmail.com. 
alone, nearly 375 million social media users represent a mass population who indulge in sharing information. More than $75 \%$ of social media users in India and the rest of the world rely on information shared on WhatsApp and other social media platforms [4]. This review focuses on the effects of social media on sharing information on nSARSCoV2 spread and COVID-19. It also highlights how false, fabricated, and manipulated information shared on different social media platforms affected lives during the pandemic.

\section{Methodology}

To address the pitfall of social media during the COVID-19 pandemic, various databases such as PubMed, PubMed Central, Web of Science, and Scientific blogs were searched for studies reporting a negative role of social media in the present COVID-19 pandemic. Different keywords such as "Pitfall", "Impact of Social Media", "Pros and Cons of Social Media", and "Scientific relevance of Social Media', in context with COVID-19 pandemic, were used. We selected articles associated with information on COVID-19 and social media as sharing platforms in each search. These articles were examined for social media issues on the COVID-19 pandemic and how it affected human lives.

\section{Results}

We found that 18 most relevant articles highlighted the negative impact of social media in sharing rumors on the cause/source of infection, disease, etiology and symptoms, preventive measures (restrictions and lockdown), guidelines for appropriate covid behavior, treatment, and vaccination.

\subsection{Social Media versus the nSARS-CoV2 spread, restrictions, and containment}

In early 2020, most of the countries across the globe were in a strict lockdown to contain the spread of the virus. In many instances, social media platforms were reported with misleading information and manipulated content. Brennen et al. demonstrated social media as a potential enemy in the current pandemic by sharing information via different types, sources, and claims of misinformation in COVID-19 [5]. It is evident that information on the global internet travel even faster than a virus and affects millions of individuals mentally, creating a panic situation. In fact, in 2020, The Lancet published an editorial "COVID- 19; fighting panic with information" [6]. The regulations associated with social media for sharing information are fragile, and in most cases, users do not abide by such regulations. The repeated and bulk amount of misleading/manipulated information creates a devastating consequence in the public domain, as seen in the current COVID- 19 pandemic [2]. The low low-quality research work often promoted via these social media platforms becomes an example of selfvalidation of shared information [7]. 


\subsection{Social media versus COVID-19 management}

The most challenging aspect of the current pandemic is the lack of treatment and an authentic source of information. In such a panic situation, a large percentage of the population relies on self-medication and seeks information on the internet. Gottlieb and Dyer demonstrated that more than 1.5 million Twitter users targeted malicious and manipulative behavior to the large population seeking self-medication in the COVID19 pandemic [7]. Malik et al. reported that social media offered non-authenticated remedies for self-medication during COVID-19 [8]. Various social media pages are filled with overwhelming information (non-authenticated scientifically) on herbal and traditional medicine [9]. More than benefits, these remedies remain associated with negative, harmful health impacts. Devastating consequences are reported in many developing countries, such as India, where a prescription is not mandatory to buy several therapeutics such as chloroquine and hydroxychloroquine [10]. The use of alternative medicine to cure disease is known since ancient times, and during COVID19 , various herbs are being used on a large scale. Here, social media platforms are the promoter for such uses. In addition, the lack of medicine and treatment has caused a large percentage of the population to find an appropriate treatment remedy not only in India but also in the rest of the world as well [11]. Several life-saving therapeutics in the case of COVID-19 are not available adequately; however, social media provides details of suppliers and sellers who are extremely unethical. There are reports that Remdesivir, a potential therapeutic for chronic COVID-19, is available for sale without prescription [12]. As we are in the middle of the pandemic, the details of life support devices such as oxygen cylinders and ventilators are readily available on social media [1]. However, in most of these social media reports, the information is either false or manipulated [13]. Additionally, there is a growing debate on the safety and efficacy of vaccines across the globe among the scientific community. There is even a new trend in social media that debates whether to take vaccines or not without any scientific basis [14].

\section{Discussion and Conclusions}

COVID-19 is the second most and worst pandemic in human history after the Spanish Flu in 1919. This pandemic is not over yet and is still affecting millions of lives daily $[15,16]$. The lack of therapeutics, life support care, and facilities has forced people to opt for alternative approaches to fight against the disease [17]. Here, social media becomes a key ally but largely an enemy as it offers non-scientific information about the diagnosis, precautions, and treatments of COVID-19 [18]. COVID-19 is not only a pandemic but also represents an unprecedented setting for the spread of misleading/manipulated information [19]. Though the first report on COVID-19 was shared on social media (Chinese social media), the role of social media has since then changed completely [15]. Different social media pages such as Facebook, YouTube, WhatsApp, Instagram, and Twitter share overwhelming information on COVID-19.

In most cases, such information is non-scientific and misleading, affecting the mental and physical health of many populations [20]. Social media is a vital tool for sharing information across the globe, but in the case of COVID-19, a large percentage of such information remains misleading and manipulated [21]. Most social media platforms have regulations related to information sharing, but these regulations are 
fragile, and users find an opportunity to violate such regulations. Therefore, there is an urgent need to find a measure to control the spread of misleading/manipulated information to help us fight against COVID-19.

\section{References}

[1] González-Padilla DA, Tortolero-Blanco L. Social media influence in the COVID-19 Pandemic. Braz J Urol. 2020 Jul 27;46:120-4.

[2] Venegas-Vera AV, Colbert GB, Lerma EV. Positive and negative impact of social media in the COVID-19 era. Rev Cardiovasc Med. 2020 Dec 30;21(4):561-564.

[3] Singh S, Dixit A, Joshi G. Is compulsive social media use amid COVID-19 pandemic addictive behavior or coping mechanism? Asian J Psychiatr. 2020 Dec;54:102290.

[4] Brennen JS, Simon F, Howard PN, Nielsen RK. Types, sources, and claims of COVID-19 misinformation. Reuters Institute. 2020 Apr 7;7(3):1.

[5] The Lancet. COVID-19: fighting panic with information. Lancet (London, England). 2020 Feb 22;395(10224):537.

[6] Gottlieb M, Dyer S. Information and Disinformation: Social Media in the COVID - 19 Crisis. Acad Emerg Med. 2020 Jul 1.

[7] Malik M, Tahir MJ, Jabbar R, Ahmed A, Hussain R. Self-medication during Covid-19 pandemic: challenges and opportunities. Drugs Ther Perspect. 2020 Dec;36(12):565-7.

[8] Chan AKM, Nickson CP, Rudolph JW, Lee A, Joynt GM. Social media for rapid knowledge dissemination: early experience from the COVID-19 pandemic. Anaesthesia. 2020 Epub ahead of print.

[9] Schillinger D, Chittamuru D, Ramírez AS. From "infodemics" to health promotion: a novel framework for the role of social media in public health. Am J Public Health. 2020 Sep;110(9):1393-6.

[10] Badell-Grau RA, Cuff JP, Kelly BP, Waller-Evans H, Lloyd-Evans E. Investigating the Prevalence of Reactive Online Searching in the COVID-19 Pandemic: Infoveillance Study. J Med Internet Res. 2020;22(10):e19791.

[11] Tsao SF, Chen H, Tisseverasinghe T, Yang Y, Li L, Butt ZA. What social media told us in the time of COVID-19: a scoping review. Lancet Digital Health. 2021 Jan 28.

[12] Abd-Alrazaq A, Alhuwail D, Househ M, Hamdi M, Shah Z. Top concerns of tweeters during the COVID-19 pandemic: infoveillance study. J Med Internet Res. 2020 Apr 21;22(4):e19016.

[13] Sumbaly R, Miller M, Shah H, Xie Y, Culatana SC, Khatkevich T, Luo E, Strauss E, Szilvasy G, Puri M, Manadhata P, Graham B, Douze M, Yalniz Z, Jegou H. Using AI to detect COVID19 misinformation and exploitative content. Facebook Artificial Intelligence. Available at: https://ai.facebook.com/blog/using-ai-to-detect-covid-19-misinformation-and-exploitative-content/.

[14] Ashton J. COVID-19 and the 'Spanish'flu. J Royal Soc Med. 2020 May;113(5):197-8.

[15] Franchini AF, Auxilia F, Galimberti PM, Piga MA, Castaldi S, Porro A. COVID 19 and Spanish flu pandemics: All it changes, nothing changes. Acta Biomed. 2020;91(2):245.

[16] Williams OD. COVID-19 and Private Health: Market and Governance Failure. Development. 2020 Dec;63(2):181-90.

[17] Al-Dmour H, Masa'deh R, Salman A, Abuhashesh M, Al-Dmour R. Influence of Social Media Platforms on Public Health Protection Against the COVID-19 Pandemic via the Mediating Effects of Public Health Awareness and Behavioral Changes: Integrated Model. J Med Internet Res. 2020;22(8):e19996.

[18] Chen E, Lerman K, Ferrara E. Tracking social media discourse about the covid-19 pandemic: Development of a public coronavirus twitter data set. JMIR Public Health Surveill. 2020 May 29;6(2):e19273.

[19] Obiała J, Obiała K, Mańczak M, Owoc J, Olszewski R. COVID-19 misinformation: Accuracy of articles about coronavirus prevention mostly shared on social media. Health Policy Technol. $2021 \mathrm{Mar}$ $1 ; 10(1): 182-6$.

[20] Naeem M. Do social media platforms develop consumer panic buying during the fear of Covid-19 pandemic. J Retail Consum Serv. 2021 Jan 1;58:102226.

[21] Anwar A, Malik M, Raees V, Anwar A. Role of Mass Media and Public Health Communications in the COVID-19 Pandemic. Cureus. 2020 Sep 14;12(9):e10453. 